\title{
Evaluation of Nephrotoxicity among First-Line Antiretroviral Therapy-Experienced Patients at the Yaounde Central Hospital
}

\author{
Kamga Wouambo Rodrigue ${ }^{1}$, Taheu Christian ${ }^{1}$, Tchatchouang Serges ${ }^{2}$ and Fokam Joseph ${ }^{3,4 *}$ \\ ${ }^{1}$ Specialized Teaching Centre for Medical Analysis Techniques (CESTAM), Cameroon \\ ${ }^{2}$ Department of Biochemistry, University of Yaounde 1, Cameroon
}

${ }^{3}$ Faculty of Medicine and Biomedical Sciences of the University of Yaounde 1, Cameroon

${ }^{4}$ Chantal BIYA International Reference Centre (CIRCB) for Research on HIV/AIDS prevention and management, Cameroon

*Corresponding author: Joseph Fokam, Faculty of Medicine and Biomedical Sciences of the University of Yaounde 1, Cameroon

\begin{tabular}{|c|}
\hline ARTICLE INFO \\
\hline Received: 慧 February 08, 2020 \\
\hline Published: February 19, 2020 \\
\hline
\end{tabular}

Citation: Kamga Wouambo Rodrigue, Taheu Christian, Tchatchouang Serges, Fokam Joseph. Evaluation of Nephrotoxicity among First-Line Antiretroviral TherapyExperienced Patients at the Yaounde Central Hospital. Biomed J Sci \& Tech Res 25(4)-2020. BJSTR. MS.ID.004248.

Keywords: Nephrotoxicity; ART; TDF; Renal Marker; HIV

\begin{abstract}
Background: The withdrawal of stavudine from the first line Antiretroviral Therapy (ART) and the introduction of tenofovir (TDF) since 2010 in sub-Saharan Africa had a direct repercussion on the initiation of many patients on this new molecule. Despite its therapeutic efficiency already proved, TDF seems to induce some side effects such as renal failures. So, it is important to study the variation of early renal marker and immunological response (CD4 cells) of patients on regimen with TDF in order to have an optimal therapeutic follow up.
\end{abstract}

Methods: A historic-prospective and longitudinal survey was carried out from September 2011 to April 2012 at the Yaounde Central Hospital including adult participants in their first 6 months of ART in compliance with ethical standards. Dosage of serum creatinine estimated Glomerular Filtration Rate and CD4 T-lymphocytes count were systematically performed at baseline and at endpoint. Besides, a urinary dipstick test was only done at endpoint. Data analysis were performed with EPI INFO 7.0 and the comparison of categorical variables were done by chi-square test. A p-value $<0.05$ was considered as statistically significant.

Results: Out of the 132 naïve and eligible patients to ART with TDF, 68.2\% were females and the mean age of the study population was 41 years old. There was an abnormal increase of creatinine from 6.1\% (D0) to 19.7\% (M6) ( $\mathrm{p}<0.01$ ) with men being more affected $(21.4 \%)$. Thirty six percent $(36 \%)$ of patients presented proteinuria at M6 and the incidence of kidney impairment was 7.6\%. A quick recovery of immune response was also observed moving from $4.8 \%$ at D0 to $25.6 \%$ at M6 ( $p<0.01)$.

Conclusion: Even though ART with TDF improves the immune response, a strict monitoring of the creatinine is recommended. We also suggested the systematical dosage of serum creatinine and other markers of renal failure among patients on TDF regimens.

\section{Introduction}

HIV/AIDS is a major public health problem worldwide with 34 millions of people affected around the world in 2011 among whom 22.9 million were in sub-Saharan African (68\%) [1]. With 2 million deaths, HIV/AIDS remains among the main cause of death in the world and the major reason of death in Africa [2]. With the "3 by 5 " initiative and the "Universal access to comprehensive Antiretroviral Therapy (ART), care and support" launched in 2005 by the global fund to fight against AIDS, the morbidity and mortality 
of HIV/AIDS have drastically decreased. Since virus discovery in 1983, many progresses have been noticed for the management of this disease. So, it moves from monotherapy (1987), then bitherapy (around 1990) and tritherapy (1996) used henceforth in the follow up and management of HIV infection. Of note, a decade of ART has transformed HIV-infection from fatal acute infection in the past to a manageable chronic disease nowadays $[3,4]$.

This treatment is essentially virostatic, reacts by enzymatic inhibition process [5] to stop all mechanisms leading to new virions and consequently increase the life expectancy of people living with HIV/AIDS. The efficiency of those ART drugs has been already proved by many scientists [6,7]. Even possible side-effects have been evaluated, leading to the elimination of stavudine from ART protocols of patient follow up in Cameroon and some molecules still have tolerable side-effects [6,7] and replaced by the Tenofovir Disoproxil Fumarate (TDF). It is a powerful reverse transcriptase nucleotidic inhibitor used in first and second line of ART. As a new molecule recently introduced in a particular context in Cameroon, the efficiency and tolerability of TDF should be evaluated. For that molecule, the monitoring of serum creatinine has been introduced among the fundamental biological exams during HIV patients follow up. TDF is known for it possible side-effects such as renal dysfunctions (nephrotoxicity, tubulopathies, acute or chronic kidneys impairment...). A survey made by scientists of an American health organization the Kaiser observed that $21 \%$ patients exposed to TDF have interrupted taking it after about 104 weeks because of a proximal renal dysfunction [8]. Its excretion is essentially kidney dependent. In fact, 70 to $80 \%$ of administered dose are found unchangeable in the urine after excretion [8]. It would be relevant to look at the responsibility of TDF in renal failures genesis that could reduce life expectancy of patients followed up in Cameroon. This evaluation of the nephrotoxicity due to TDF will give a better appreciation of renal filtration rate of patients henceforth on this new molecule in our country. This information is important to improve the life quality expectancy hampered by possible sideeffects of ART. This survey is for a public health interest, not only for our country Cameroon but also for the other sub-regional countries which have also introduced TDF in their national ART guidelines.

\section{Methods}

\section{Study Design and Population}

A historical-prospective and longitudinal survey was conducted from September $15^{\text {th }} ; 2011$ to April 30 ${ }^{\text {th }}, 2012$ at Yaounde Central Hospital. This hospital was selected as the study sentinel site based on its long term-experience on patients management with ART, its conformity to the national ART guidelines, and its ability to enroll at least 132 patients on ART in a 6 months period. So, 132 naïves HIV-infected patients were enrolled and followed-up during their 6 first months of ART. The study participants were consecutively enrolled at their follow-up period based on inclusion criteria (aged $>15$ years old; eligible for enrollment on first line ART at the study site; not previously enrolled on ART, and not being transferred from another HIV clinic). This cohort was monitored for 6 months after treatment initiation on a first line ART regimen with TDF, with an additional study period of three months for the recruitment and monitoring of lost to follow-up cases. A standardized questionnaire was administered to assess demographic, epidemiological, clinical, treatment, and adherence information at month zero $\left(M_{0}\right)$ and month six $\left(M_{6}\right)$ after treatment initiation. At enrollment on ART $\left(\mathrm{M}_{0}\right)$, lymphocytes TCD4 and a dosage of serum creatinine were performed while at M6, a urinary dipstick test was added.

\section{Sample Processing}

Following questionnaire administration, samples were processed for serum creatinine and CD4 count were performed before ART initiation at M0 and at M6 in the immunology and virology laboratories of the hospital. A total of $10 \mathrm{ml}$ whole blood was collected from each study participant in 2 tubes. The EDTA tube was used for lymphocytes TCD4 count and dry tube for creatinemia. Deaths were recorded and patients were considered lost to follow-up if they were not seen at the hospital for longer than 9 months after starting ART. At M6, the urine of each participant was collected in a sterile box for urinary dipstick test.

\section{Follow Up Procedures}

TCD4 Lymphocytes Count: It was performed using the CYFLOW Counter ${ }^{\circledR}$ (2 parameters) and the BD FASCOUNT (4 parameters). The immunological response was defined by the increase of TCD4 lymphocytes rates of study participant according to the WHO recommendations.

Dosage of Creatinine and Estimation of Glomerular Filtration Rate: Serum creatinine concentration was measured on Ryato ${ }^{\circledR}$ analyser with TECO Diagnostic reagent. Quality control of chemistry analyzers was performed according to manufacturer recommendations. An estimation of glomerular filtration rate (eGFR) was calculated using the modification diet in renal disease (MDRD study equation) which estimates creatinine clearance on the basis of the age, serum creatinine and the race instead of CockroftGault formulas which includes serum creatinine, weight and gender [9] or chronic kidney disease-epidemiology (CKD-EPI) formula, the most modern, accurate but recent. This surrogate measure of eGFR has been validated in sub-Saharan Africa [10]. eGFR was categorized according to the U.S. National Kidney Foundation's Kidney Disease Outcome Quality Initiative (K/DOQI) as normal ( $\geq 90 \mathrm{ml} / \mathrm{min}$ ), mild $(60-89 \mathrm{ml} / \mathrm{min})$, moderate $(30-59 \mathrm{ml} / \mathrm{min})$ and severe $(<30 \mathrm{ml} / \mathrm{min})$ renal dysfunction [11]. There were no patients in our analysis with severe renal dysfunction at baseline.

Urinary Dipstick Test Processing: A midstream urine of each participant was collected in a sterile and clean container. The removed urine was then applied to the sterile dipstick and the urine test trip (Medi-test combi 10) was performed according to manufacturer recommendations. Suprapubic aspiration with a 
fresh sterile syringe or fresh catheter, not always practical, wasn't used in our context because no patient was confine to bed. So, each of them was then able to provide fresh urine. Ten tests on each strip was detected (blood, ketones, glucose, $\mathrm{pH}$, bilirubin, urobilinogen, protein, leukocytes, nitrite and specific gravity).

Statistical Analysis: Data were analyzed through Epi info 7.0. The comparison of categorical variables was done using chi-square test and student t-test was used for mean comparisons. Quantitative variables were reported as means and/or medians while qualitative variables were reported in percentage. P-value less than 0.05 was considered as statistically significant.

Ethical Considerations: Ethics approval was obtained from the National Ethics Committee ( $n^{\circ}$ 235/CNE/SE/2011) of Cameroon and an authorization of research was delivered by the director of Central Hospital of Yaounde. At the patient's recruitment site, an information sheet was administered to all eligible patients, who then provided their informed consent prior to enrollment into the study. Informed consent was verbal, rather than written, because patients were monitored as per the national antiretroviral therapy program guidelines (i.e. during their normal consultation appointments). Since our aim was to evaluate the nephrotoxicity due to ART, verbal consent was the most applicable approach in minimizing a change in patient's routine behavior/adherence during the study period, and in identifying the routine functionality (i.e. strengths and weaknesses) of the ART program at the studied site. Thus, informed consent was documented in the patient's medical files throughout the study. This consent procedure was approved by the ethics committee. Confidentiality was secured by the use of unique identification codes attributed to each of the study participants. Because all the participating patients were $>21$ years old (i.e. majority age in Cameroon) without any other vulnerability, informed consent from parents or legal representative was not applicable in our study.

\section{Results}

\section{Characteristics of Study Population}

Out of the 132 HIV patients enrolled, $68.2 \%$ were females with a sex ratio male/female of $1 / 2$. The median of the study population was 41 years old [22 to 68 years] and they were all naïves and eligibles to first line ART with TDF. The mean of TCD4 at the baseline was 291 cells [ 1 to 657 cells]. The majority of participants (79.5\%) was under TDF+3TC+EFV (TENLAM-E) while 20.5\% were under TDF+3TC+NVP (TENLAM-N). The serum creatinine was normal $(<13 \mathrm{mg} / \mathrm{l})$ among $93.9 \%$ of participants. Subjects with an eGFR> $5050 \mathrm{ml} / \mathrm{min} / 1.73 \mathrm{~m} 2$ were the most represented [88.6\%, $\mathrm{n}=117$ ].

\section{Evolution of Renal Marker}

At M6, an increase of abnormal creatinemia see Table 1 was noticed $(6.1 \%$ at $\mathrm{M} 0$ to $19.7 \%$ at $\mathrm{M} 6, \mathrm{p}<0.01)$ and a proteinuria of $36.4 \%(42 / 132)$ in general population $(21.4 \%$ males versus $18.9 \%$ females, $\mathrm{p}=0.117$ ) The incidence of kidney impairment was estimated at $7.6 \%$. The age group [37; 53 [ seemed to be the most affected with $5.3 \%(7 / 132)$ at M0 with eGFR<50 versus $11.36 \%$ (15/132) at M6 see Table 2. At $M_{6}$, a considerable number of study participants was affected by a risk of nephrotoxicity especially females $(22.2 \%)$ versus $11.9 \%$ of men $(5 / 42)$ but the difference was not statistically significant $(\mathrm{p}=0.15)$.

Table 1: Creatinemia of study participants.

\begin{tabular}{|c|c|c|c|c|c|c|c|c|c|}
\hline & & \multicolumn{3}{|c|}{$M_{0}$} & \multicolumn{5}{|c|}{$\mathbf{M}_{6}$} \\
\hline \multicolumn{2}{|c|}{ Gender } & \multicolumn{2}{|c|}{ Male } & \multicolumn{2}{|c|}{ Female } & \multicolumn{2}{|c|}{ Male } & \multicolumn{2}{|c|}{ Female } \\
\hline \multicolumn{2}{|c|}{ Number (n) and percentage (\%) } & $\mathrm{n}$ & $\%$ & $\mathrm{n}$ & $\%$ & $\mathrm{n}$ & $\%$ & $\mathrm{n}$ & $\%$ \\
\hline \multirow{2}{*}{ Creatinemia } & Normal & 37 & 88.1 & 87 & 96.7 & 33 & 78.6 & 73 & 81.1 \\
\hline & Abnormal & 5 & 11.9 & 3 & 3.3 & 9 & 21.4 & 17 & 18.9 \\
\hline \multicolumn{2}{|c|}{ Total } & 42 & 100 & 90 & 100 & 42 & 100 & 90 & 100 \\
\hline
\end{tabular}

Note: $\mathrm{M}_{0}$ : Day 0; $\mathrm{M}_{6}$ : Month 6.

Table 2: Estimated Glomerular Filtration Rate of study participants.

\begin{tabular}{|c|c|c|c|c|c|c|c|c|c|c|c|c|c|c|}
\hline & & & \multicolumn{6}{|c|}{$\mathbf{M}_{0}$} & \multicolumn{6}{|c|}{$\mathbf{M}_{6}$} \\
\hline \multicolumn{3}{|c|}{ Age group } & \multicolumn{2}{|c|}{ ] 21;37[ } & \multicolumn{2}{|c|}{$[37 ; 53[$} & \multicolumn{2}{|c|}{$[53 ; 69[$} & \multicolumn{2}{|c|}{ ] 21;37[ } & \multicolumn{2}{|c|}{$[37 ; 53[$} & \multicolumn{2}{|c|}{$[53 ; 69[$} \\
\hline \multicolumn{3}{|c|}{ Gender } & M & $\mathrm{F}$ & M & $\mathrm{F}$ & M & $\mathrm{F}$ & M & $\mathrm{F}$ & M & $\mathrm{F}$ & $\mathrm{M}$ & $\mathrm{F}$ \\
\hline & & $\mathrm{n}$ & 7 & 33 & 19 & 36 & 14 & 8 & 7 & 33 & 17 & 30 & 13 & 7 \\
\hline & $\geq 50$ & $\%$ & 16.7 & 36.7 & 45.2 & 40 & 33.3 & 8.9 & 16.7 & 36.7 & 40.5 & 33.3 & 31 & 7.8 \\
\hline \multirow[t]{2}{*}{ GFR } & & $\mathrm{n}$ & 0 & 4 & 1 & 6 & 1 & 3 & 0 & 4 & 3 & 12 & 2 & 4 \\
\hline & $<50$ & $\%$ & 0 & 4.4 & 2.4 & 6.7 & 2.4 & 3.3 & 0 & 4.4 & 7.1 & 13.3 & 4.8 & 4.4 \\
\hline
\end{tabular}

Note: GFR: Glomerular Filtration Rate (ml/min per 1.73 m2); M: Male; F: female; $M_{0}$ : Day 0; $M_{6}$ : Month 6; n: number. 


\section{Immune Response Recovery}

An increase of subjects with TCD4 $\geq 500$ (4.8\% to $25.6 \%$ ) was noticed whereas a net reduction of severe immune depressives subjects TCD $4<200$ (38.4\% to 13.6\%) was also observed (see Table 3). The mean of TCD 4 after 6 months of ART was 476 cells (78 to 1001 cells).

Table 3: T-CD4 cell rates of 125 subjects.

\begin{tabular}{|c|c|c|c|c|}
\hline & \multicolumn{2}{|c|}{$\mathrm{M}_{0}$} & \multicolumn{2}{c|}{$\mathrm{M}_{6}$} \\
\hline CD4 cell rate & $\mathrm{n}$ & $\%$ & $\mathrm{n}$ & $\%$ \\
\hline Absent $(>500)$ & 6 & 4,8 & 32 & 25.6 \\
\hline Moderate (349-499) & 25 & 20 & 39 & 31.2 \\
\hline advanced (200-349) & 46 & 36.8 & 37 & 29.6 \\
\hline Severe (<200) & 48 & 38.4 & 17 & 13.6 \\
\hline Total & 125 & 100 & 125 & 100 \\
\hline
\end{tabular}

Note: $\mathrm{M}_{0}$ : Day 0; $\mathrm{M}_{6}$ : Month 6; CD4: Cluster of Differentiation; n: number

\section{Discussion}

In this cohort done in 6 months in Yaounde (Cameroon) on HIV-1 eligibles patients on a first line ART regimen with TDF, the mean age of study population was 41 years with a predominance of females (68.2\%). These results are similar to other studies. A study on 212 participants done at the University Health Centre in Yaounde (Cameroon), showed that $72.6 \%$ of study participants were females versus $27.4 \%$ of males [12]. More, at the Mutengene Baptist Health Centre located in the in the South West Region of Cameroon reported $73.2 \%$ females (212/337) [13]. Indeed, women are the most affected by HIV in Cameroon with a ratio of 170 women infected per 100 men (6.8\% versus 4.1\%) [14].

At $\mathrm{M}_{6}$, an abnormal increase of creatinemia cases was observed ( 8 at M0 to 26 at $M_{6}$ ) with $21.4 \%$ for males vs $18.9 \%$ for females $(\mathrm{p}=0.73)$. This increase of serum creatinine in patients under TDF was also reported earlier in USA [8]. In fact, serum creatinine is influenced by gender, age and muscle mass. However, formulas that predict GFR take into account gender, age and weight but not muscle mass $[9,15]$. Besides, $36.4 \%(n=48)$ subjects had a positive semi-quantitative urine dipstick test. A cohort of 107 patients in 6 months in Montpellier (France) found 22\% of proteinuria [16]. That difference between high proteinuria in comparison to creatinemia (in $\mathrm{M}_{6}$ ) could be due to a probable renal failure start that must be investigated. Regular measurement of kidney function in HIV-infected individuals at presentation and throughout TDF use is essential $[17,18]$. The new South African ART guidelines recommend measurement of serum creatinine and creatinine clearance at ART initiation, at 3 and 6 months and yearly thereafter [19].

After 6 months of ART, the incidence of kidney impairment $(\mathrm{GFR}<50)$ of patients aged more than 37 years was 7,6\% $(n=10)$ and those patients were already in need of an adjustment of TDF dosage as recommended in case of eGFR decrease. Alternatively, those patients could be switched to zidovudine-lamivudineefavirenz/névirapine [19]. The studies carried out in California, in Maryland, in Virginia and in Washington between January 2002 and 2006 among 964 naïve patients under TDF have found same results with an incidence of $8 \%$ of renal proximal impairment [20]. Furthermore, the best access to ART brings out a serious problem the aging process of people living with HIV/AIDS, because the physiological loss of nervous cells is associated to decrease of eGFR in adults of about $0.5-1 \mathrm{ml} / \mathrm{min} / 1.73 \mathrm{~m} 2$ per year [21]. Such an increase of $7.6 \%$ of chronic kidney impairment among people living with HIV/AIDS getting aged in about 6 months reinforce the idea of the strict surveillance of creatinemia on this patient initiated on TDF in Cameroon as in another country like south Africa [19].

For immune response, at M0, $75.2 \%$ of subjects had a CD4 count $<350$ with $38.4 \%$ less than 200 cells and $36.8 \%$ between 200 and 350 cells. Only $4.8 \%$ of the study participants had a CD4 count $\geq 500$. The mean of CD4 at initiation was 291 cells. Similar data has been found in Zurich in 2005 [22]. The high value of immunedepressives subjects (75.4\%) as well as the mean CD4 at $\mathrm{M}_{0}$ could be explained by the eligibility criteria for ART initiation, the financial difficulties to good health care services. Many positives screened subjects disappeared and generally returns to the hospital in pitiful conditions needing urgently an ART. In fact, according to WHO recommendations 2010 it is admitted that patients with T-CD $4<350$ cells/mm3 should be directly eligible to ART [8]. Thus, a significant regression of severe immune-depressives subjects $(C D 4<200)$ and an increase of subjects with $\mathrm{CD}^{3} 500$ ( $4.8 \%$ at $\mathrm{M}_{0}$ to $25.6 \%$ at $\mathrm{M}_{6}$ ) were reported at M6. It reflects the good immunological response of patients under TDF protocol, a powerful nucleotide inhibitor of reverse transcriptase.

\section{Conclusion}

This study revealed a high incidence of renal failure in TDF regimens. Even if ART with TDF increases the immune response, a strict monitoring and follow up of the main kidney biochemical marker such as creatinine, proteinuria should be regularly done. A similar survey done on a very large-scale population would be very interesting. Furthermore, an exploration of TDF toxicity (kidney, bone ...) in a pediatrics context would be relevant for the practical routine.

\section{Disclosure Statement}

The authors declare no conflicts of interest.

\section{Acknowledgement}

Our gratitude goes towards participants and the staff of University Health Centre in Yaounde.

\section{Funding}

Not applicable. 


\section{References}

1. (2011) UNAIDS: How to get to zero: Faster, Smarter, Better. In.: United Nations Programme on HIV/AIDS.

2. (2009) UNAIDS: Joint Action for Results: UNAIDS outcome framework, 2009-2011. In.: Joint United Nations Programme on HIV/AIDS.

3. (2003) WHO: The WHO and UNAIDS global initiative to provide antiretroviral therapy to 3 million people with HIV/AIDS in developing countries by the end of 2005 In.: World Health Organization.

4. Deeks SG, Lewin SR, Havlir DV (2013) The end of AIDS: HIV infection as a chronic disease. Lancet 382(9903): 1525-1533.

5. Yoly V, Rachine A (2003) New antiretrovirals, antibiotics. Rev du Prat 5: 77-82.

6. Fokam J, Salpini R, Santoro MM, Cento V, Perno CF, et al. (2011) Drug resistance among drug-naive and first-line antiretroviral treatmentfailing children in Cameroon. The Pediatric infectious disease journal 30(12): 1062-1068.

7. Kouanfack C (2009) Access to antiretroviral treatment in Africa: The case of Cameroon. Montpellier: University of Montpellier I.

8. Horberg M, Tang B, Towner W, Silverberg M, Bersoff-Matcha S, et al. (2010) Impact of tenofovir on renal function in HIV-infected, antiretroviral-naive patients. Journal of acquired immune deficiency syndromes 53(1): 62-69.

9. Cockcroft DW, Gault MH (1976) Prediction of creatinine clearance from serum creatinine. Nephron 16(1): 31-41.

10. Sanusi AA, Akinsola A, Ajayi AA (2000) Creatinine clearance estimation from serum creatinine values: Evaluation and comparison of five prediction formulae in Nigerian patients. African journal of medicine and medical sciences 29(1): 7-11.

11. (2002) K/DOQI clinical practice guidelines for chronic kidney disease: Evaluation, classification, and stratification. American journal of kidney diseases: The official journal of the National Kidney Foundation 39(2 Suppl 1): S1-266.

\section{ISSN: 2574-1241}

DOI: $10.26717 /$ BJSTR.2020.25.004248

Fokam Joseph. Biomed J Sci \& Tech Res

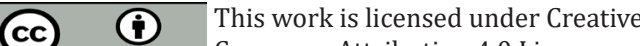
Commons Attribution 4.0 License

Submission Link: https://biomedres.us/submit-manuscript.php
12. Salpini R, Fokam J, Ceccarelli L, Santoro MM, Nanfack A, et al. (2016) High Burden of HBV-Infection and Atypical HBV Strains among HIVinfected Cameroonians. Current HIV research 14(2): 165-171.

13. Gachara G, Magoro T, Mavhandu L, Lum E, Kimbi HK, et al. (2017) Characterization of occult hepatitis B virus infection among HIV positive patients in Cameroon. AIDS research and therapy 14(1): 11.

14. (2008) UNGASS: Implementation of the Declaration of Commitment on HIV/AIDS. In. Edited by Drugs UNGASSo. Cameroon: United Nations General Assembly Special Session on Drugs.

15. Lamb EJ, Tomson CR, Roderick PJ (2005) Estimating kidney function in adults using formulae. Annals of clinical biochemistry 42(Pt 5): 321-345.

16. Peyriere H, Cournil A, Casanova ML, Badiou S, Cristol JP, et al. (2015) Long-Term Follow-Up of Proteinuria and Estimated Glomerular Filtration Rate in HIV-Infected Patients with Tubular Proteinuria. PloS one 10(11): e0142491.

17. Fabian J, Naicker S (2009) HIV and kidney disease in sub-Saharan Africa. Nature reviews Nephrology 5(10): 591-598.

18. Clumeck N, Pozniak A, Raffi F (2008) European AIDS Clinical Society (EACS) guidelines for the clinical management and treatment of HIVinfected adults. HIV Med 9(2): 65-71.

19. (2010) National Department of Health, Africa RoS: The South African Antiretroviral. Treatment Guidelines. 2010. Available online. In. South Africa: South African National Department of Health.

20. (2011) Washington Uo: Pivotal study finds that HIV medications are highly effective as prophylaxis against HIV infection in men and women in Africa. In. Edited by Washington Uo.

21. Meier P, Dayer E, Donzé N (2009) Read the markers of the function and their value predictors. Caduceus Express 11(9).

22. Maitland D, Moyle G, Hand J, Mandalia S, Boffito M, et al. (2005) Early virologic failure in HIV-1 infected subjects on didanosine/tenofovir/ efavirenz: 12-week results from a randomized trial. AIDS (London, England) 19(11): 1183-1188.

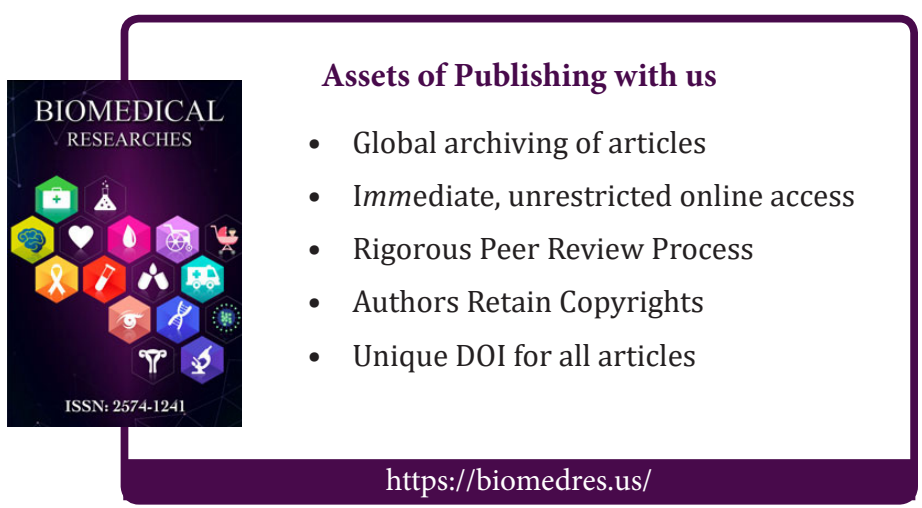

